\title{
A CONVERSE TO THE LOG-LOG LAW FOR MARTINGALES
}

\author{
Dedicated to the memory of Hanna Neumann \\ W. L. STEIGER \\ (Received 1 June 1972, revised 29 October 1973) \\ Communicated by M. F. Newman
}

\begin{abstract}
For sums of independent and identically distributed random variables $x_{n}$, the HartmanWintner law of the iterated logarithm is equivalent to $x_{n} \in L_{2}$. We show that this is also true when the $x_{n}$ form a stationary, ergodic martingale difference sequence. This is accomplished by extending a theorem of Volker Strassen to the present contexi.
\end{abstract}

\section{Introduction}

In this paper we consider a sequence $\left\{x_{j}\right\}$ of identically distributed random variables on a probability space $(\Omega, \mathscr{A}, p)$ and the following two conditions:

$$
\begin{gathered}
x_{n} \in L_{2} \text { and } E\left(x_{n}\right)=0, E\left(x_{n}^{2}\right)=K^{2} \text { for some } K, \\
p\left\{\limsup _{n \rightarrow \infty}\left(x_{1}+\cdots+x_{n}\right) /(2 n \log \log n)^{\frac{1}{2}}=T^{2}\right\}=1 \text { for some } T .
\end{gathered}
$$

As usual, $E$ denotes Lebesgue integration with respect to $p$ of $\mathscr{A}$-measurable functions on $\Omega$. For fixed $t>0, L_{t}$ is the set of functions $f$ on $\Omega$ for which $E\left(|f|^{t}\right)<\infty$, while for $A \in \mathscr{A}$, " $A$ a.e." means $p(A)=1$.

If the $x_{n}$ are independent, (A) implies (B), with $T^{2}=K^{2}$, by the celebrated Hartman-Winter law of the iterated logarithm [2]. In fact (B) implies (A), with $K^{2}=T^{2}$, as shown by Strassen [6], who thus elucidated the true nature of the $\log$ - log law for sums of independent, identically distributed random variables. Like the central limit theorem $\left(\left(x_{1}+\cdots+x_{n}\right) /\left(n K^{2}\right)^{\frac{1}{2}}\right.$ converges in law to the unit normal distribution if and only if (A)), it is a second order result.

Even without independence such a characterization of the law of the iterated logarithm may obtain. Specifically, suppose that the $x_{n}$ form a martingale difference sequence with respect to an increasing sequence $(\phi, \Omega)=\mathscr{F}_{0} \subseteq \cdots$ $\subseteq F_{n} \subset \cdots$ of sigma sub-algebras of $A$; i.e., $x_{n} \in L_{1}$ is $\mathscr{F}_{n}$ measurable and 
$E\left(x_{n+1} \mid \mathscr{F}_{n}\right)=0$ a.e., all $n$, where $E\left(\cdot \mid \mathscr{F}_{j}\right)$ denotes a version of the conditional expectation operator on the $\mathscr{F}_{j}$ measurable functions. Then if the $x_{n}$ are also stationary and ergodic the Hartman-Wintner theorem extends and (A) still implies (B), as was shown by Stout [5]. Of course, $E\left(x_{n}\right)=0$ is redundant in (A).

Interestingly, Strassen's theorem extends too, and (B) still implies (A), as will be shown in the next section. The law of the iterated logarithm remains a second order result in the martinglale case.

\section{The result}

We prove the following statement.

THEOREM. Let $\left\{x_{j}\right\}$ be a stationary and ergodic sequence of random variables on a probability space $(\Omega, \mathscr{A}, p)$. If $\left\{x_{j}, \mathscr{F}_{j}\right\}$ is a martingale difference sequence and if condition (B) holds, then also condition (A) holds with $K^{2}=T^{2}$.

Proof. Without loss of generality we may take $\Omega$ to be the set of all extended real valued sequences $\left\{\cdots, u_{-1}, u_{0}, u_{1}, \cdots\right\}$, the $x_{j}$ to be the coordinate random variables on $\Omega, \mathscr{A}$ to be the $\sigma$-algebra generated by the $x_{j}, p$ to be the probability generated by finite dimensional distributions, and $\mathscr{F}_{j}$ to be the $\sigma$-field generated $\left\{x_{k},-\infty<k \leqq j\right\}$. A standard construction assures that there is such a representation that preserves the original finite dimensional distributions, so that the given stochastic structure is not changed.

It suffices to prove $x_{n} \in L_{2}$ since $E\left(x_{n}^{2}\right)=T^{2}$ then follows directly from Stout's theorem. Also, the distribution of $x_{i}$ may be taken as continuous since the distribution of $y_{n}=x_{n}+u_{n}$ is (the $u_{n}$ are independent uniform random variables on $[-1,1]$ and are independent of the $x_{n}$ ) and because the $y_{n}$ form a stationary, ergodic martingale difference sequence and satsfy (i) $y_{n} \in L_{2}$ if and only if $x_{n} \in L_{2}$; (ii) $\lim \sup \left(y_{1}+\cdots+y_{n}\right) /(2 n \log \log n)^{\frac{1}{2}}$ is finite a.e. if and only if $\lim \sup \left(x_{1}+\cdots+x_{n}\right) /(2 n \log \log n)^{\frac{1}{2}}$ is.

By way of contradiction, suppose that (B) holds while $x_{n} \notin L_{2}$. Using ideas from [3], fix $T>0$ and choose numbers $C>0, D<0$ so that, writing $J$ $=[D, C]$, the following conditions hold:

$$
\begin{gathered}
E\left(x_{n} \mid x_{n} \in J\right)=0 \\
E\left(x_{n}^{2} \mid x_{n} \in J\right)>8 T^{2} \\
y=p\left\{x_{n} \in J\right\}>\frac{1}{2}
\end{gathered}
$$

Define the random variables $U_{n}=I\left(\left\{x_{n} \in J\right\}\right)$ and $V_{n}=I\left(\left\{x_{n} \notin J\right\}\right)$ where, for $A \in \mathscr{A}, I(A)$ is the function from $\Omega$ to $R$ taking values 1 when $\omega \in A$ and 0 when $\omega \notin A$. As both are measurable functions of $x_{n}$, the ergodic theorem applies to show that

$$
\left(U_{-j}+\cdots+U_{0}+\cdots+U_{k}\right) /(j+k+1) \rightarrow y
$$


a.e. and

$$
\left(V_{-j}+\cdots+V_{0}+\cdots+V_{k}\right) /(j+k+1) \rightarrow 1-y
$$

a.e., both as $j+k \rightarrow \infty . E\left(x_{n}^{2}\right)=\infty$ means $y<1$ so that by (3), both $\sum_{l=-j}^{k} U_{l}$ and $\sum_{l=-j}^{k} V_{l} \rightarrow \infty$ as $j+k \rightarrow \infty$; the import of these statements is that $p\left\{x_{n} \in J\right.$ for infinitely many $n \in M\}=p\left\{x_{n} \notin J\right.$ for infinitely many $\left.n \in M\right\}=1$, where $M$ is any infinite set of consecutive integers. Define (random) subsequences $\{\lambda(j)\}$ and $\{\mu(j)\}$ by.

$\lambda(1)=\min \left(n>0: x_{n} \in J\right)$

$\lambda(j+1)=\min \left(n>\lambda(j): x_{n} \in J\right)$

$\lambda(j)=\max \left(n<\lambda(j+1): x_{n} \in J\right)$

$$
\mu(1)=\min \left(n>0: x_{n} \notin J\right)
$$$$
\mu(j+1)=\min \left(n>\mu(j): x_{n} \notin J\right), j>0
$$$$
\mu(j)=\max \left(n<\mu(j+1): x_{n} \notin J\right), j \leqq 0 .
$$

By the above remarks, the sequences are well-defined, except possibly on a null set; similarly with the sequences $\left\{Y_{j}\right\}$ and $\left\{Z_{j}\right\}$ defined by

$$
Y_{n}=x_{\lambda(n)} \text { and } Z_{n}=x_{\mu(n)}
$$

Let $G_{n}$ be the $\sigma$-subalgebra of $A$ generated by $\left\{Y_{i},-\infty<i \leqq n\right\}$, define $t_{n}^{2}=E\left(Y_{n}^{2} \mid G_{n-1}\right)$ and, for $m \geqq 1, u_{m}^{2}=t_{1}^{2}+\cdots+t_{m}^{2}$. Observe that the recurrence times $\left\{\delta_{j}\right\}, \delta_{n}=\lambda(n)-\lambda(n-1)$ are stationary since the $x_{j}$ are, and accordingly, $\left\{Y_{j}\right\}$ is also stationary, a fact which easily gives the stationarity of $\left\{t_{j}^{2}\right\}$.

Now $Y_{n}$ is $G_{n}$-measurable and $E\left(Y_{n} \mid G_{n-1}\right)=0$, a.e. Because $\left|Y_{n}\right| \leqq C-D$ a.e. and $p\left\{\sum_{n=1}^{\infty} t^{2}=\infty\right\}>0$, the conditions for Stout's [4] martingale analogue of the Kolmogoroff log-log law are satisfied. Accordingly,

$$
p\left\{\limsup _{n \rightarrow \infty}\left(Y_{1}+\cdots+Y_{n}\right) /\left(2 u_{n}^{2} \log \log u_{n}^{2}\right)^{\frac{1}{2}}=1\right\}>0 .
$$

Using the stationarity of $\left\{t_{j}^{2}\right\}$, the ergodic theorem and (2) imply that for any $\delta>0, p\left\{u_{n}^{2} \geqq 8 n T^{2}\right\}>1-\delta$ for all sufficiently large $n$. Again by the ergodic theorem, $p\left\{Z_{1}+\cdots+Z_{\lambda(n)-n} \geqq 0\right.$ for infinitely many $\left.n>0\right\}>0$. Finally $x_{1}+\cdots+x_{\lambda(n)}=\left(Y_{1}+\cdots+Y_{n}\right)+\left(Z_{1}+\cdots+Z_{\lambda(n)-n}\right)$ for $n>0$, by (4). These facts combine with (5) to establish

$$
p\left\{\limsup _{n \rightarrow \infty}\left(x_{1}+\cdots+x_{\lambda(n)}\right) /\left(8 n T^{2} \log \log n\right)^{\frac{1}{2}} \geqq 1\right\}>0 .
$$

Finally, by (3) and the ergodic theorem, $p\{\lambda(n) \geqq 2 n$ for only finitely many $n>0\}=1$ so that from (6)

$$
p\left\{\limsup _{n \rightarrow \infty}\left(x_{1}+\cdots+x_{\lambda(n)}\right) /\left(3 \lambda(n) T^{2} \log \log \lambda(n)\right)^{\frac{1}{2}} \geqq 1\right\}>0,
$$

a contradiction to (B) that completes the proof.

REMARK. We conclude by pointing out a related problem that remains open. If the $x_{n}$ are independent, (B) is equivalent to $\left(x_{1}+\cdots+x_{n}\right) /\left(n T^{2}\right)^{t}$ converging in 
law to the unit normal distribution, i.e., the central limit theorem. With the result of the preceding section, the martingle central limit theorem (see [1], e.g.) would be equivalent to the $\log \log$ law if the former result entailed $x_{n} \in L_{2}$.

\section{Acknowledgement}

I want to thank R.W. Shorrock for many cogent remarks and the referee for suggestions that improved the clarity.

\section{References}

[1] P. Billingsly, 'The Lindeberg-Lévy theorem for martingale', Proc. Amer. Math. Soc. 12 (1961), 788-792.

[2] P. Hartman and A. Wintner, 'On the law of the iterated logarithm', Amer. J. Math. 63 (1941), 169-176.

[3] W. L. Steiger and S. K. Zaremba, 'The converse of the Hartman-Wintner theorem', $Z$. Wahrscheinlickeitstheorie verw. Geb. 22 (1972), 193-194.

[4] W. F. Stout, 'A martingale analogue of Kolmogorov's law of the iterated logarithm', $Z$. Wahrscheinlichkeitstheorie verw. Geb. 15 (1970), 279-290.

[5] W. F. Stout, 'The Hartman-Wintner law of the iterated logarithm for martingales', Ann. Math. Statist. 41 (1970), 2158-2160.

[6] V. Strassen, 'A converse to the law of the of the iterated logarithm', $Z$. Wahrscheinlichkeitstheorie verw. Geb. 4 (1966), 265-268.

Centre de Recherches Mathématiques

Université de Montréal

Canada 